\title{
Change in renal function post-nephrectomy for renal cell carcinoma in patients with and without hypertension and/or diabetes
}

\author{
J John, ${ }^{1,2}$ M Henry, ${ }^{3}$ A Ringoir, ${ }^{2}$ G Pinto, ${ }^{2}$ K Kesner, ${ }^{1}$ J Lazarus, ${ }^{2}$ S Sinha ${ }^{2}$ \\ ${ }^{1}$ Department of Urology, Walter Sisulu University and Frere Hospital, South Africa \\ ${ }^{2}$ Division of Urology, Department of Surgery, University of Cape Town and Groote Schuur Hospital, South Africa \\ ${ }^{3}$ Centre for Higher Education Development, University of Cape Town, South Africa
}

Corresponding author, email: jeffveenajohn@gmail.com

Background: The standard of care for surgically resectable disease renal cell carcinoma (RCC) is a nephrectomy. Post nephrectomy, these patients are at risk for the development of new onset chronic kidney disease or the progression of pre-existing chronic kidney disease. We aimed to report the changes in renal function in patients who had a nephrectomy for RCC.

Methods: This retrospective, descriptive, cross-sectional study identified 137 patients who had a nephrectomy for RCC from 1 January 2009 to 31 December 2017. The pre-nephrectomy and post-nephrectomy estimated glomerular filtration rate (eGFR) and the histological subtype of RCC on histopathological analysis of the resected specimen were recorded from the National Health Laboratory Services online results platform. All analyses were conducted using SPSS (Version $25)$ and the significance level was set at $p<0.05$.

Results: After a mean follow-up period of $26.5 \pm 22$ months (median $=19$ months), the patients' eGFR dropped by a mean of $4.82 \pm 8.67 \mathrm{ml} / \mathrm{min} / 1.73 \mathrm{~m}^{2}$ (95\% CI 3.23-6.41) post-nephrectomy. The mean eGFR fall in patients' who had hypertension and/or diabetes $(n=63)$ was significantly larger compared to patients who had neither of these comorbidities $\left(n=54 ; p<.001 ;\right.$ mean $=7.30 \pm 8.40 \mathrm{ml} / \mathrm{min} / 1.73 \mathrm{~m}^{2}(95 \%$ CI $5.19-9.42)$ and $1.93 \pm 8.14 \mathrm{ml} / \mathrm{min} / 1.73 \mathrm{~m}{ }^{2}(95 \% \mathrm{CI}$ $0.30-4.15)$ respectively.

Conclusions: The decline in renal function in patients with hypertension and/or diabetes mellitus is more pronounced than in patients with neither of these comorbidities. In these high-risk patients, measures must be taken to prevent the development and limit the progression of chronic kidney disease.

Keywords: RCC, Nephrectomy, eGFR, CKD

\section{Background}

Renal cell carcinoma (RCC) is the thirteenth most common type of malignancy globally, accounting for $3.3 \%$ of all newly diagnosed cancers. ${ }^{1}$ Globally, 342000 new kidney cancer cases are diagnosed annually. ${ }^{2}$ In the United States alone, the American Cancer Society's most recent estimate for kidney cancer for 2018 was approximately 63340 new cases of kidney cancer. ${ }^{3}$ The most recent available figures from South Africa (SA) show that 602 cases of RCC were diagnosed histologically in $2013 .{ }^{4}$

With the population aging and widespread availability of cross-sectional abdominal imaging techniques, the absolute incidence of renal cancer shows an increasing trend over the last few years. ${ }^{5}$

For patients with surgically resectable disease, the standard of care for RCCs is a nephrectomy. Post nephrectomy, these patients are at risk for the development of new onset chronic kidney disease (CKD) or the progression of preexisting CKD. As a consequence, these patients are at risk of increased cardiovascular disease (CVD) and all-cause mortality. Renal dysfunction activates pathways which leads to endothelial dysfunction and atherosclerosis with subsequent increases in CVD. This increased risk of CVD was initially thought to be limited to patients with end stage renal disease (ESRD). However, it has now been shown that there is an increased risk of CVD across all stages of CKD. The United States Renal Data System 2014 annual report stated that the prevalence of any cardiovascular disease is double in patients with CKD $(69.8 \%$ vs $34.8 \%) .{ }^{6}$ The risk of cardiovascular mortality has been shown to increase by $5 \%$ for every $10 \mathrm{~mL} / \mathrm{min}$ per $1.73 \mathrm{~m}^{2}$ reduction in estimated glomerular filtration rate (eGFR). ${ }^{7}$ Controversy exists about whether all patients are at risk for the development and progression of CKD, post nephrectomy, or if it is just patients with significant risk factors.

We aimed to report the changes in renal function, based on the eGFR in patients who had a nephrectomy for RCC at Groote Schuur Hospital, over a nine-year period.

\section{Methods}

This was a retrospective, descriptive, cross-sectional study performed at Groote Schuur Hospital (GSH), Cape Town, South Africa. During the study period (1 January 2009 to 
31 December 2017), 137 patients who had a nephrectomy for RCC were identified through a departmental operative database. Due to incomplete data, twenty patients were excluded from the study $(n=117)$. Demographic data and the presence of hypertension and/or diabetes were recorded from the hospital folder records. The pre-nephrectomy and post-nephrectomy eGFR and the histological subtype of $\mathrm{RCC}$ on histopathological analysis of the resected specimen were recorded from the National Health Laboratory Services (NHLS) online results platform. The pre-nephrectomy renal function was defined as the eGFR immediately prior to surgery. The post-nephrectomy results were defined as the nadir eGFR at least 3 months post-nephrectomy. The eGFR was calculated using the abbreviated Modification in Diet and Renal Disease (MDRD) study equation, i.e. GFR $=186$ $\times(\text { serum creatinine })^{-1.154} \times(\text { age })^{-0.203}$, from which the result is multiplied by 0.742 for female patients. Laboratories, including the NHLS, do not report the actual MDRD eGFR values $>60 \mathrm{ml} / \mathrm{min} / 1.73 \mathrm{~m}^{2}$ range as there is a significant underestimation of eGFR (increased false positive diagnosis of CKD). ${ }^{8}$ As a result, the highest eGFR recorded was 60 $\mathrm{ml} / \mathrm{min} / 1.73 \mathrm{~m}^{2}$. Hypertension was defined as self-reported hypertension with or without ongoing pharmacologic treatment. Diabetes mellitus was defined as a history of self-reported diabetes mellitus treated pharmacologically or controlled by diet.

\section{Statistical analysis}

Descriptive statistics were explored and are presented as means and standard deviations for continuous variables, and proportions for categorical variables. An independentsample $t$-test was used to determine if there was a difference in the change in eGFR between those with hypertension and/ or diabetes and those without. Pearson correlations assessed the relationship between change in eGFR and predictor variables. Thereafter, a backwards linear regression analysis was used to determine which factors predicted change in eGFR function. All analyses were conducted using SPSS (Version 25) and the significance level was set at $p<0.05$.

\section{Results}

The mean age of the population was $57 \pm 12$ years. Sixtyseven $(57 \%)$ were males. On histology, 74 patients $(63 \%)$ had clear cell RCC (ccRCC), 27 (23\%) had papillary RCC (pRCC), 9 (7.7\%) had chromophobe RCC (crRCC), $2(2 \%)$ had an unclassified histology, and $5(4 \%)$ had other rarer histology. The mean eGFR at baseline did not differ significantly between those with hypertension and/ or diabetes and those without $(p=0.061)$. After a mean follow-up period of $26.5 \pm 22$ months (median $=19$ months), the patients' eGFR dropped by a mean of $4.82 \pm$ $8.67 \mathrm{ml} / \mathrm{min} / 1.73 \mathrm{~m}^{2}(95 \% \mathrm{CI} 3.23-6.41)$ post-nephrectomy.
The median eGFR fall in patients who had hypertension and/or diabetes $(n=63)$ was significantly larger compared to patients who had neither of these comorbidities $(n=54$; $p<.001) ;$ median $=-7 \mathrm{ml} / \mathrm{min} / 1.73 \mathrm{~m}^{2}(\mathrm{IQR}=-13-0)$ and 0 $\mathrm{ml} / \mathrm{min} / 1.73 \mathrm{~m}^{2}(\mathrm{IQR}=-0.25-0)$ respectively $)-($ Figure 1$)$.

Only the predictors age and the presence of hypertension and/or diabetes significantly correlated with change in eGFR (Table 1). These two variables were entered into a backwards linear regression. When entered into the regression and accounting for hypertension and/or diabetes, age no longer predicted change in eGFR $(p=.128)$. However, having hypertension and/or diabetes significantly predicted change in eGFR $(p=.003)$ and explains $7 \%$ of the variance in change in eGFR. On average, having hypertension and/or diabetes predicated a $4.76 \mathrm{ml} / \mathrm{min} / 1.73 \mathrm{~m}^{2}$ greater decline in eGFR than patients without hypertension and/or diabetes. Therefore, it appears that only the presence of hypertension and/or diabetes is a predictor of change in eGFR.

\section{Discussion}

The relationship between RCC and CKD has been described as bi-directional. Increasing age, hypertension, diabetes, smoking, obesity and analgesic abuse are common risk factors for the development of both RCC and CKD. The high prevalence of pre-nephrectomy CKD among those with RCCs reflects common risk factors in these patients. ${ }^{9}$ $\mathrm{CKD}$ is a risk factor for the development of acquired renal

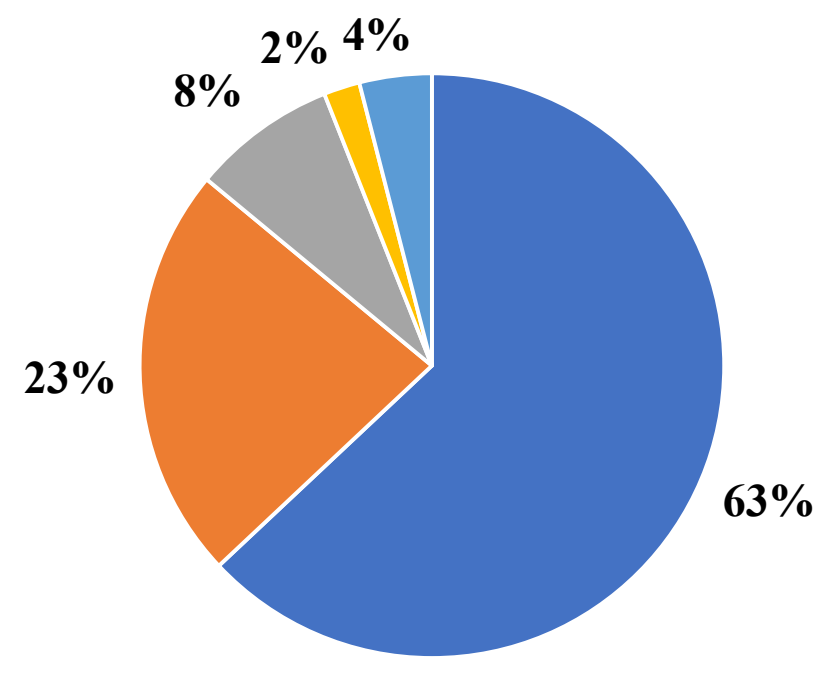
- Clear Cell RCC
- Chromophobe RCC
- Papillary RCC
- Unclassified RCC

Figure 1: Distribution of the histological subtypes of renal cell carinoma

Table 1: Correlations between outcome and predictor variables

\begin{tabular}{lccccc}
\hline & Change eGFR & Age & Gender & HPT/DM & eGFR risk \\
\hline Change eGFR & 1.00 & $-.214^{*}$ & .015 & $-.280^{*}$ & -.076 \\
Age & & 1.00 & $.184^{*}$ & $.282^{*}$ & $.348^{*}$ \\
Gender & & 1.00 & .039 & .156 \\
HPT/DM & & & 1.00 & $.265^{*}$ \\
eGFR risk & & & & 1.00
\end{tabular}

Note: Pearson's $r$ correlation coefficients are presented $p<.05$

HPT - hypertension; DM - diabetes mellitus; eGFR - estimated glomerular filtration rate 


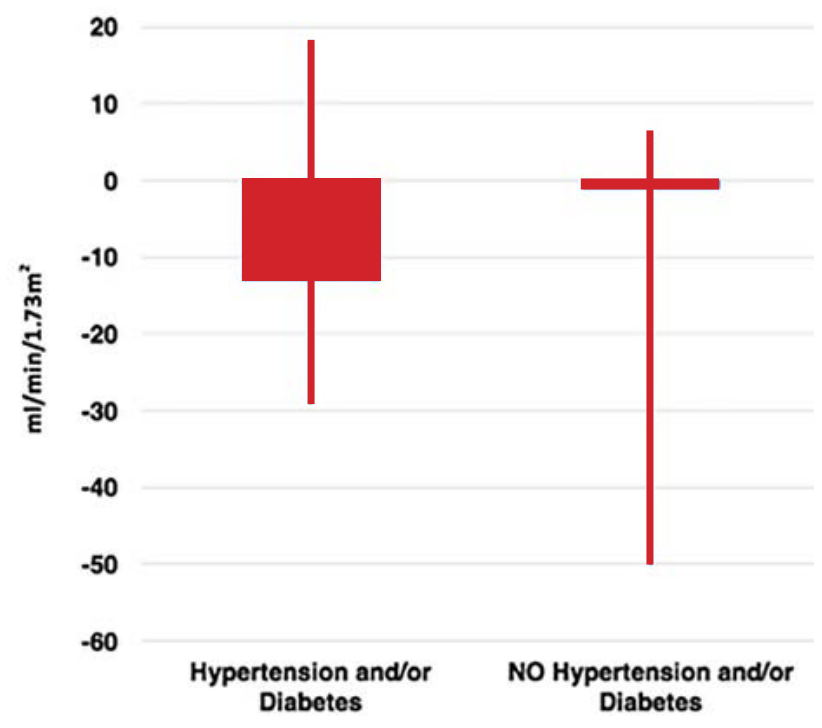

Figure 2: Change in eGFR

cysts, which in itself is a risk factor for RCC. In addition, post-nephrectomy, the loss of functional renal tissue and ischaemia at time of nephrectomy and the chronic hyperfiltration driven partly by an increase in glomerular pressure compromises renal function post-surgery. ${ }^{10}$

In the present study, we evaluated eGFR to determine postoperative changes in renal function in patients with and without hypertension and/or diabetes. The mean eGFR decline post-nephrectomy in patients who had hypertension and/or diabetes is significantly larger compared to patients who had neither of these comorbidities (Figure 2). Our findings were corroborated by other reports in the literature.

Brandina et al. reported that hypertension $(\mathrm{OR}=3.7)$ and diabetes mellitus $(\mathrm{OR}=11.6)$, amongst others, were significant predictors of renal function loss after surgery. ${ }^{11}$ After a multivariate logistic regression, glomerulosclerosis $(p=0.039)$ and baseline eGFR (0.01) were independent predictors for new onset CKD. In addition, Charlson Comorbidity Index (0.011), which is a method of predicting mortality by grading comorbidities, was also an independent predictor for new onset CKD. The pre-nephrectomy eGFR was measured within 30 days before surgery, and the post-nephrectomy at last follow-up (at least 6 months after surgery). There are some limitations that need to be considered for this trial. They initially started with 222 patients who underwent radical nephrectomy between 1999 and 2006 at Sírio Libanes Hospital (São Paulo, Brazil). However, the final cohort consisted of only 65 patients. Only patients who had nephrectomy specimens available for histopathological analysis were included in the study. ${ }^{11}$

A univariate analysis by Jeon et al. showed that the presence of both diabetes $(p<.006)$ and hypertension $(p<.006)$ were associated with CKD after surgery. The median time to the development of CKD with and without diabetes was 13 and 21 months respectively. ${ }^{12}$

Apart from hypertension and diabetes, other risk factors have been identified for new-onset CKD or acceleration of pre-existing CKD after radical nephrectomy. Acute kidney injury (AKI) in the immediate post-surgical setting is a significant risk factor for the development of CKD. Risk factors for the development of AKI in the postoperative setting include advanced age, male gender, increased body mass index and decreased pre-nephrectomy eGFR. Cho et al. reviewed the eGFR in patients a year post radical nephrectomy and reported the median eGFR to be lower in the group that developed AKI post-nephrectomy when compared to those without AKI, highlighting the need for clinicians to ensure AKI is prevented post-nephrectomy. ${ }^{13}$ Other predisposing factors resulting in CKD postnephrectomy include advanced age, pre-nephrectomy renal dysfunction and the presence of adjacent non-neoplastic kidney parenchyma abnormalities including arteriosclerosis, interstitial fibrosis and glomerulosclerosis. ${ }^{12}$ The College of American Pathologists has recommended reporting of nonneoplastic renal parenchyma of all tumour nephrectomy specimens. ${ }^{14}$ The basis of this recommendation is that studies have shown that arterionephrosclerosis and glomerular diseases frequently coexist with RCC. ${ }^{15}$

The impact of worsening renal function and higher rates of CKD in patients post-nephrectomy cannot be overlooked. It has been associated with increased risk of metabolic and cardiovascular diseases and subsequent mortality. Weight et al. reported that the loss of renal function observed with radical nephrectomy was associated with a $25 \%$ increased risk of cardiovascular-related mortality and a $17 \%$ increased risk all-cause mortality on multivariate analysis. ${ }^{16}$ Renal function compromise causes activation of the reninangiotensin system, oxidative stress, inflammation with increased levels of cytokines, and dyslipidaemia. This leads to endothelial dysfunction and atherosclerosis with subsequent increases in risk of heart failure, coronary artery disease and cerebrovascular accidents. ${ }^{16,17}$

While this study shows a significant decline in eGFR in patients with hypertension and/or diabetes, it also highlights that the eGFR decline in patients with neither of these risk factors may not be as pronounced as previously thought. Preoperatively patients with increased risk of eGFR decline should be identified and referred to the nephrology unit, in order to assess and discuss the loss of renal function, the consequences thereof and potential risk of starting haemodialysis in future. Intraoperatively, nephron sparing surgery should be attempted where possible, without compromising oncological outcome, and ischaemia time must be kept to a minimum. In the immediate postoperative setting, it is imperative that measures be taken to prevent postoperative AKI. In the long term, all patients must be carefully followed up to detect reductions in eGFR sooner to facilitate early appropriate evaluation and intervention for CKD. Improved coordination of care among urologists, pathologists, and nephrologists will result in better outcomes for RCC patients.

Some limitations of the present study should be considered. Ideally, a renal scan should be used to measure and quantify renal function. ${ }^{19}$ However, this technique is not routinely used in clinical practice. A common surrogate for renal function is the measurement of the creatinine clearance and estimation equations based upon serum creatinine. The use of serum creatinine as a marker of renal function is a limitation in itself as it is influenced substantially by muscle mass, hydration status, body habitus, and food contents. ${ }^{20}$ In addition, the NHLS and other laboratories, do not routinely report the actual MDRD eGFR values $>60 \mathrm{ml} / \mathrm{min} / 1.73 \mathrm{~m}^{2}$ range due to a significant underestimation of eGFR. ${ }^{8}$ The highest eGFR we recorded was $60 \mathrm{ml} / \mathrm{min} / 1.73 \mathrm{~m}^{2}$. We acknowledge changes in patients with a eGFR $>60 \mathrm{ml} / \mathrm{min} / 1.73 \mathrm{~m}^{2}$ may have been 
missed. Secondly, even though the study is based in a single institution, it is a retrospective analysis of data, and biases of selection exist. In addition, we lacked data on recognised confounding factors, such as smoking, obesity and analgesic abuse. The present study lacked a specific, standardised post-nephrectomy time period for the evaluation of eGFR. Lastly, it is the nature of a retrospective record review that data captured was heavily reliant on hospital folder records. We lacked data on the grade of hypertension, type of diabetes and the duration that our patients had these diagnoses before surgery. Nevertheless, the present study is the first in a South African setting highlighting the concerns of deteriorating renal function post nephrectomy. More studies with larger sample sizes and longer follow-ups with serial determinations of renal functions are needed to validate the results of the present study.

\section{Conclusion}

There are long standing concerns over the significant deterioration of renal function, post nephrectomy and this was reaffirmed in our setting. The decline in renal function in patients with hypertension and/or diabetes mellitus is more pronounced than in patients with neither of these comorbidities. In these high-risk patients, measures must be taken to prevent the development and limit the progression of CKD.

\section{Conflict of interest}

The authors declare that there is no conflict of interest.

\section{Funding source}

Funding from the Discovery Foundation is gratefully acknowledged.

\section{Ethical approval}

Ethics approval was obtained from the University of Cape Town Faculty of Health Sciences Human Research Ethics Committee (HREC REF 465/2018).

\section{REFERENCES}

1. Ferlay J, Soerjomataram I, Dikshit R, et al. Cancer incidence and mortality worldwide: Sources, methods and major patterns in GLOBOCAN 2012. Int J Cancer. 2014;136(5):E359-E386. https://doi.org/10.1002/ijc.29210.

2. Global Burden of Disease Cancer Collaboration. Global, regional, and national cancer incidence, mortality, years of life lost, years lived with disability, and disability-adjusted life-years for 29 cancer groups, 1990 to 2016: A systematic analysis for the Global Burden of Disease Study. JAMA Oncol. 2018;4(11):1553-68. https://doi.org/10.1001/ jamaoncol.20 18.2706.

3. Siegel R, Miller K, Jemal A. Cancer statistics, 2018. CA Cancer J Clin. 2018;68(1):7-30. https://doi.org/10.3322/ caac. 21442 .

4. National Health Laboratory Services. Cancer in South Africa 2013: Full report. National Cancer Registry. 2013. Available from: http://www.nioh.ac.za/wp-content/ uploads/2018/03/2013NCR.pdf. Accessed 16 April 2019.

5. Znaor A, Lortet-Tieulent J, Laversanne M, et al. International variations and trends in renal cell carcinoma incidence and mortality. Eur Urol. 2015;67(3):519-30. https://doi. org/10.1016/j.eururo.2014.10.002.
6. Subbiah A, Chhabra Y, Mahajan S. Cardiovascular disease in patients with chronic kidney disease: a neglected subgroup. Heart Asia. 2016;8(2):56-61. https://doi.org/10.1136/ heartasia-2016-010809.

7. Manjunath G, Tighiouart H, Coresh J, et al. Level of kidney function as a risk factor for cardiovascular outcomes in the elderly. Kidney Int. 2003;63(3):1121-9. https://doi. org/10.1046/j.1523-1755.2003.00838.x.

8. Naiker J. Glomerular filtration rate (GFR) and estimation of the GFR (eGFR) in adults. Continuing Medical Education. 2012;30(7):235-7.

9. Choi SK, Song C. Risk of chronic kidney disease after nephrectomy for renal cell carcinoma. Korean J Urol. 2014;55(10):636-42. https://doi.org/10.4111/ kju.2014.55.10.636.

10. Perazella M, Dreicer R, Rosner M. Renal cell carcinoma for the nephrologist. Kidney International. 2018;94(3):471-83. https://doi.org/10.1016/j.kint.2018.01.023.

11. Brandina R, Moreira K, Gregório E, et al. Histologic abnormalities in non-neoplastic renal parenchyma and the risk of chronic kidney disease following radical nephrectomy. Urology. 2017;100:158-62. https://doi.org/10.1016/j.urology. 2016.09.041.

12. Jeon H, Jeong I, Lee J, et al. Prognostic factors for chronic kidney disease after curative surgery in patients with small renal tumors. Urology. 2009;74(5):1064-8. https://doi.org/ 10.1111/j.1464-410X.2011.10531.x.

13. Cho A, Lee J, Kwon G, et al. Post-operative acute kidney injury in patients with renal cell carcinoma is a potent risk factor for new-onset chronic kidney disease after radical nephrectomy. Nephrol Dial Transplant. 2011;26(11):3496501. https://doi.org/10.1093/ndt/gfr094.

14. Srigley J, Amin M, Delahunt B, et al. Protocol for the examination of specimens from patients with invasive carcinoma of renal tubular origin. College of American Pathologists. Arch Pathol Lab Med. 2010;134(4):25-30. https://doi.org/10.1043/1543-2165-134.4.e25.

15. Bijol V, Mendez G, Hurwitz S, Rennke H, Nose V. Evaluation of the nonneoplastic pathology in tumor nephrectomy specimens: Predicting the risk of progressive renal failure. Am J Surg Pathol. 2006;30(5):575-84. https://doi.org/10.1097/01. pas.0000194296.74097.87.

16. Weight C, Larson B, Fergany A, et al. Nephrectomy induced chronic renal insufficiency is associated with increased risk of cardiovascular death and death from any cause in patients with localized cT1b renal masses. J Urol. 2010;183(4):131723. https://doi.org/10.1016/j.juro.2009.12.030.

17. Go A, Chertow G, Fan D, McCulloch C, Hsu C. Chronic kidney disease and the risks of death, cardiovascular events, and hospitalization. N Engl J Med. 2004;351(13):1296-305. https://doi.org/10.1056/NEJMoa041031.

18. Anavekar N, McMurray J, Velazquez E, et al. Relation between renal dysfunction and cardiovascular outcomes after myocardial infarction. N Engl J Med. 2004;351:1285-95. https://doi.org/10.1056/NEJMoa041365.

19. Shirasaki Y, Tsushima T, Saika T, Nasu Y, Kumon H. Kidney function after nephrectomy for renal cell carcinoma. Urology. 2004;64(1):43-7. https://doi.org/10.1016/j.urology. 2004.02.039.

20. Patel SS, Molnar MZ, Tayek JA, et al. Serum creatinine as a marker of muscle mass in chronic kidney disease: results of a cross-sectional study and review of literature. J Cachexia Sarcopenia Muscle. 2013;4(1):19-29. https://doi.org/10.1007/ s13539-012-0079-1. 